\title{
A Simple Energy-based Approach of Stent Elastic Properties
}

\author{
Shuai Wang ${ }^{1, *}$, Kaifa Zhou ${ }^{2}$ and Zhong $\mathrm{Li}^{2}$ \\ ${ }^{1}$ College of Intelligent Manufacturing, Chongqing Vocational College of Transportation, 7 Xuefu Road, Jiangjin District Chongqing , \\ China \\ ${ }^{2}$ School of Civil Engineering, Chongqing Jiaotong University, 66 Xuefu Avenue, Nanan District, Chongqing, China
}

\begin{abstract}
Simple closed-form expressions were derived for the elastic moduli of lozenge grid structure based on the convenient beam theory and energy conservation principle. Finite element analysis was employed to validate the analytical estimates of the Young's modulus. The theoretic results were also compared with the numerical data in the literature. The results show that the calculation method of Young's modulus obtained by energy conservation is feasible, which provides a new way for stress analysis of sandwich structures. At the same time, the cell structure proposed in this paper provides a new scheme for the design of vascular stent.
\end{abstract}

\section{Introduction}

At present, stenting is a common method for the treatment of cardiovascular and cerebrovascular diseases. It supports the vessel wall by placing medical stents in the stage of coronary artery stenosis to keep the blood flow unblocked. Therefore, the research on the performance of vascular stents has become a hot topic. In this paper, a lozenge grid structure with negative Poisson's ratio aiming to get the large deformation is proposed in this paper. Meanwhile, the equivalent Young's modulus and Poisson's ratio of this structure are analyzed in the range of linear elasticity, and the analytical formula are achieved. Moreover, the relation between equivalent elastic parameters and geometric variables is also studied.

There were a lot of reelevating works being done before. Davood Mousanezhada[1] used finite element method to simulate PTCA surgery. The results were consistent with the actual data. It was the first time to show the role of finite element method in the treatment of vascular stenosis. In order to study the expansion characteristics of stents, Brauer carried out experiments of balloon dilatation in vitro on stents with different materials and designs, established a stent-balloon model to simulate the expansion process of stent. In the later research, a large number of simulations to the expansion process of stent were carried out. Etave[2], Migliavacca[3,4], Chua[5]put forward their own simulation methods of stent expansion. However, they didn't take the role of balloon into consideration, and the pressure was directly loaded to the inner surface of the stent model to expand it.

On its fatigue, and thought that the conventional fatigue theory was not suitable for the study of the stent fatigue. Perry[11] used finite element method to study the fatigue characteristics of the stent, and considered that it was necessary to use finite element method to study the fatigue of the bracket.

\section{Equivalent Young's modulus calculation of lozenge grid structure}

\subsection{Lozenge grid structure geometry}

A representative unit cell of the lozenge grid structure configuration shows in Figure 1. The parameters L, R, r, $\alpha$ and $t$ represent the rod length, the radius and the thickness of the rod, respectively. The depth of the cell $b$ is not shown for clarity. Structure parameters in detail are shown in Table 1.

\subsection{Analytical formulation}

In this paper, a new negative Poisson's ratio structurelozenge grid structure- is proposed. We will derive closed-form expressions for the equivalent elastic moduli of lozenge grid structure as a demonstration of our proposed method. The detailed derivations for the structure are presented in Fig. 1.

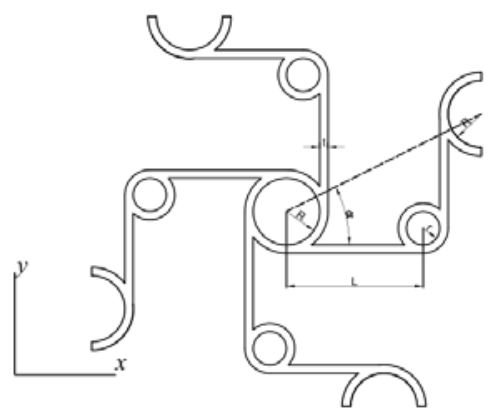

Fig.1. Cell Unit.

Corresponding author: ws15993500427@163.com 
Table 1. Cell unit sizes.

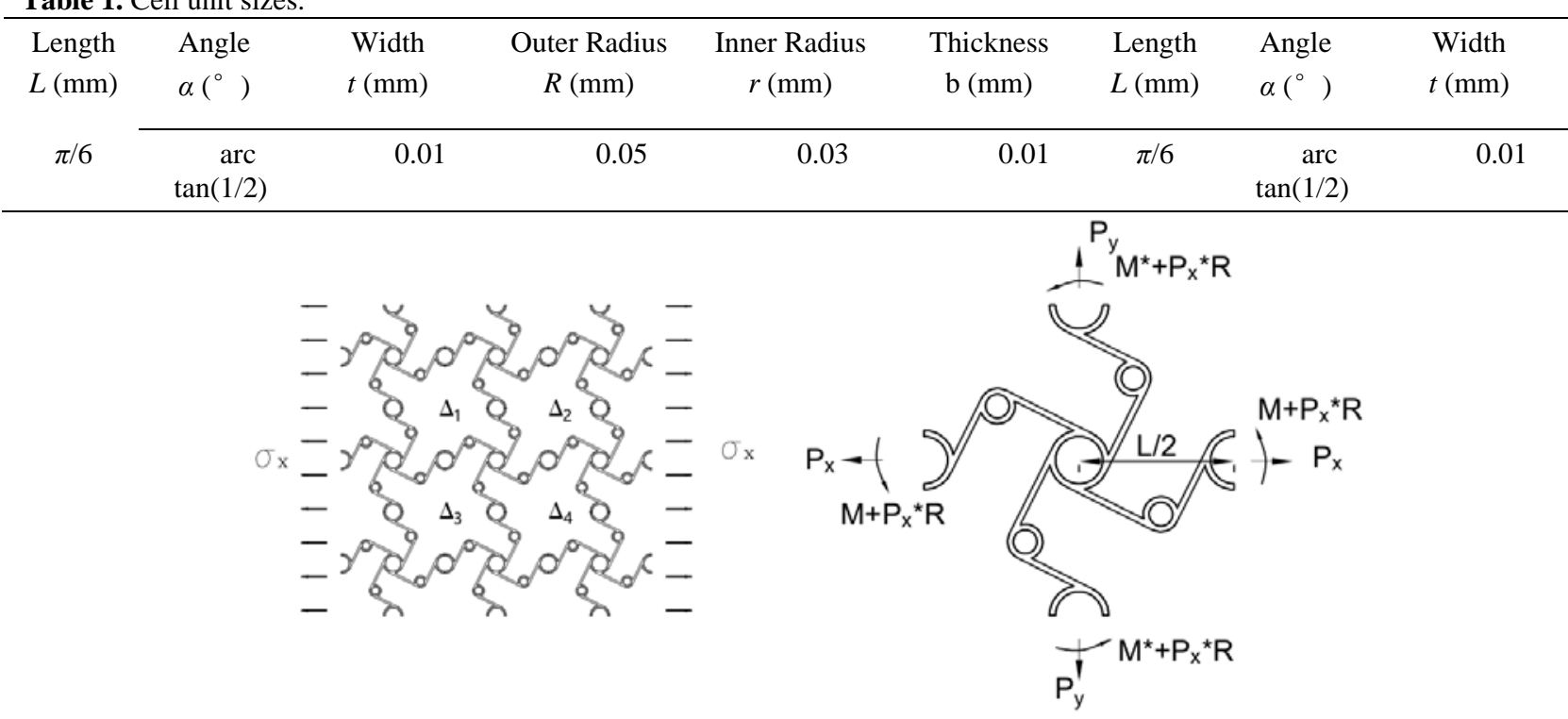

A

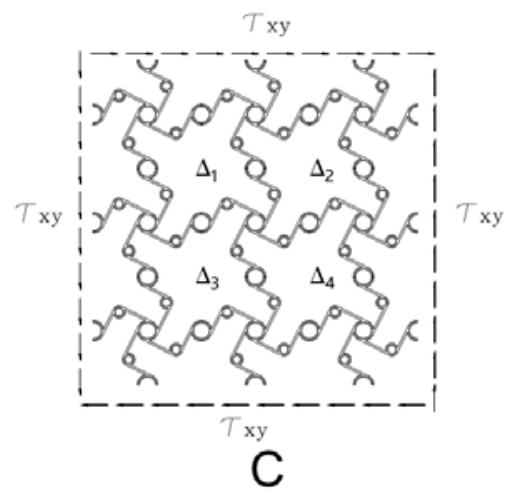

B

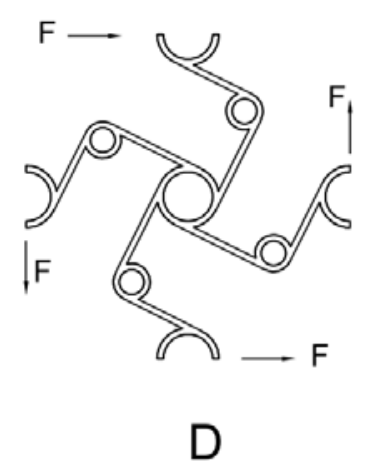

Fig.2. (a) Schematic of lozenge grid structure under x-direction axial loading. (b) Free body diagram of the structural unit cell under axial loading. (c) Schematic of lozenge grid structure under x-y shearing load. (d) Free body diagram of the structural unit cell under shearing load.

A schematic of a lozenge grid structure, which is based on an underlying square network, bears a uniaxial stress in the x-direction, $\sigma \mathrm{x}$ is shown in Fig. 2(a). The cell unit force diagram is shown in Fig. 2(b). The unit cell contains five cylinders with radius $\mathrm{R}$, four cylinders with radius $r$ and four half ligaments. which are assumed to be rigid. Due to the $180^{\circ}$ rotational symmetry of the structure and the components of the microscopic stresses, all four external cut points of the unit cell must be moment-free under an arbitrary macroscopic stress state; $\tau x y$ is shown in Fig. 2(c). Also, since the only far-field stress acting on the structure is along the $\mathrm{x}$-direction, all the external cut points must be force-less except points 1 and 2 which carry a pair of forces with opposite directions along the $\mathrm{x}$-direction due to $\sigma \mathrm{x}$. Therefore, unknown forces and moments acting on the unit cell's external cut points can be summarized as shown in Fig.2(b), where Px can be determined as a function of applying stress as:

$$
P_{X}=\sigma_{X}\left(L^{2}+(R-r)^{2}\right)^{1 / 2}
$$

To calculate equivalent elastic parameters of the proposed structure, a mechanical analysis model has been proposed based on the following assumptions:

(1) The nodes in structural configurations are perfectly rigid rotating units, and only small deformations occur within the rod.

(2) The Young's modulus and Poisson's ratio can be derived for lozenge grid structure isotropic honeycomb making use of conventional beam theory.

(3) The circle is considered as rigid body.

Moreover, to calculate the structure's Poisson's ratio, a pair of virtual forces are employed. Py is also added on points 2 and 4 of the unit cell. The strain energy of the unit cell is given as: 


$$
\begin{aligned}
& U=2 \frac{P_{x}^{2}(L+R+r)}{2 E_{s} A}+2 \frac{P_{y}^{2}(L+R+r)}{2 E_{s} A}+4 \int_{0}^{L+r} \frac{(x F)^{2}}{2 E_{s} I} d x+2 \frac{\left(\left(P_{x}-F\right) / 2\right)^{2}(L / 2+R)}{2 E_{s} A}+2 \frac{\left(\left(P_{y}-F\right) / 2\right)^{2}(L / 2+R)}{2 E_{s} A} \\
& +4 \int_{0}^{L+r} \frac{\left(x\left(P_{x}-F\right) / 2+F L^{2} /(3 L+2 R)+F(L / 2+R)^{2}\right.}{2 E_{s} I} d x+4 \int_{0}^{r} \frac{\left(x\left(P_{y}-F\right) / 2+F L^{2} /(3 L+2 R)+F(L / 2+R)^{2}\right.}{2 E_{s} I} d x
\end{aligned}
$$

where Es is the Young's modulus of the cell material, $\mathrm{A}$ is the cross sectional area of cell walls (i.e., for a rectangular cross section with unit depth, $A=t$ ), $I$ is the second moment of area of the wall's cross section (cell walls are assumed to have a rectangular cross section with uniform thickness $t$, and unit depth, i.e., $I=t^{3} / 12$, and $\alpha=\tan ^{-1}[(R-r) / L]$ is the angle between each ligament and the line connecting the centers of two adjacent cylinders as shown in Fig. 1.

Next $\partial \mathrm{U} / \partial \mathrm{Px} \mid \mathrm{Py}=0, \mathrm{~F}=0$ gives the total displacement of point 1 with respect to point 3 in the

$\mathrm{x}$-direction as:

$\delta$ 11=9LPx(4AEs)-1+2rPx (AEs)-1+5PxR(2AEs)-1

+L3Px(3IEs)-1+L2Pxr(IEs)-1 + LPxr2(IEs)-1 +

r3Px(3IEs)-1

From this, we can now calculate the structure's average strain in the $\mathrm{x}$-direction:

$$
\epsilon 11=\delta 11(2 \mathrm{~L}+2 \mathrm{R}+2 \mathrm{r})-1
$$

The Young's modulus of the structure normalized by material's Young's modulus is then defined as the ratio of the average stress $\sigma 11$ and the average strain $\epsilon 11$ :

E11Es-1 =

4t(16L3+48L2r+48Lr2+16r3+9Lt2+8rt2+10Rt2) -1

Similarly, the Young's modulus of the structure distributed along the other directions are as followed:

E22 Es-1=2Lt3((L+r+R)(16r3+8rt2+(9L+10R) t2 )) -1

On the other hand, $\partial \mathrm{U} / \partial \mathrm{F} \mid \mathrm{Px}=0$, Py $=0$ gives the structure's shear modulus with respect to the $\mathrm{x}-\mathrm{y}$ coordinate system. Then, the shear modulus of the structure normalized with respect to the Young's modulus of cell wall material, is defined as the ratio of the averages hear stress, $\tau$ xy to the average shear strain, $\gamma$ xy and given as the following:

\section{E33 Es-}

$1=(\mathrm{L}(3 \mathrm{~L}+2 \mathrm{R}) 2 \mathrm{t} 3)((\mathrm{L}+\mathrm{r}+\mathrm{R})(600 \mathrm{~L} 5+24 \mathrm{~L} 4(6 \mathrm{r}+66 \mathrm{R})+\mathrm{L} 3(3$ $60 \mathrm{r} 2+3648 \mathrm{rR}+1984 \mathrm{R} 2+9 \mathrm{t} 2)+8 \mathrm{R} 2(24 \mathrm{r} 3-$

48r2R+96rR2+Rt2)+6L2(72r332r2R+688rR2+224R3+5 Rt2)+4LR(144r3-216r2R+672rR2+96R3+7Rt2))) -1 (7)

After further derivation, the angular strain of principal stress in other directions is obtained.

$$
\varepsilon_{12}=\varepsilon_{21}=0
$$

So, the Poisson's ratio is $v_{12}=v_{21}=-\frac{\varepsilon_{21}}{\varepsilon_{11}}=0$.

The Young's moduli of the structure distributed along the other directions are as followed:

E13 Es-1 $=((2 \mathrm{~L}(3 \mathrm{~L}+2 \mathrm{R}) \mathrm{t} 3))(((\mathrm{L}+\mathrm{r}+\mathrm{R})(72 \mathrm{~L} 4+32 \mathrm{~L} 3$ $(3 \mathrm{r}+5 \mathrm{R})-3 \mathrm{~L} 2(8 \mathrm{r} 2-96 \mathrm{rR}-32 \mathrm{R} 2+\mathrm{t} 2)+4 \mathrm{R}(-8 \mathrm{r} 3+24 \mathrm{r} 2 \mathrm{R}-$ Rt2)-8L(6r3-12r2R-24rR2+Rt2))) -1

E31 Es-1 $=((2 \mathrm{~L}(3 \mathrm{~L}+2 \mathrm{R}) \mathrm{t} 3))(((\mathrm{L}+\mathrm{r}+\mathrm{R})(72 \mathrm{~L} 4+32 \mathrm{~L} 3$ $(3 \mathrm{r}+5 \mathrm{R})-3 \mathrm{~L} 2(8 \mathrm{r} 2-96 \mathrm{rR}-32 \mathrm{R} 2+\mathrm{t} 2)+4 \mathrm{R}(-8 \mathrm{r} 3+24 \mathrm{r} 2 \mathrm{R}-$ Rt2)-8L(6r3-12r2R-24rR2+Rt2))))-1

E23 Es-1=2L(3L+2R)t3((L+r+R)(3L2(40r2-t2)+4R(8r3+24r2R-Rt2)-8L(6r3-24r2R+Rt2)))-1

E32 Es-1 =-4(3L+2R)t3(3L2 (-40r2+t2)+8L(6r3$24 r 2 R+R t 2)+4 R(8 r 3-24 r 2 R+R t 2))-1$

\section{FEM Simulations and Discussion}

\subsection{FEM Simulations and Material Parameters}

In this paper, COMSOL Multiphysics computing platform is used for finite element simulation. In order to simulate the real working conditions, the material adopts self-defined material parameters and its parameters are in Table 2. And the depth of stent is $0.03 \mathrm{~mm}$.

Table 2. Test parameters.

\begin{tabular}{lrlrlr}
\hline Item & \multicolumn{5}{c}{ Parameters(mm) } \\
\hline Radius & 0.05 & Radius & 0.05 & Radius & 0.05 \\
\hline Length & $\pi / 8$ & Length & $\pi / 8$ & Length & $\pi / 8$ \\
\hline
\end{tabular}

Free tetrahedron method is used in mesh generation. The maximum cell size is $0.3 \mathrm{~mm}$, the minimum cell size is $0.01 \mathrm{~mm}$, and the maximum cell growth rate is 1.5 . The total number of cells is 1128 . Finally, the non-linear large plastic strain solver was used to calculate the plastic strain of the stent during expansion.

\subsection{Comparison between theory and FEM}

Tensile tests were carried out on the structure by applying symmetrical constraints on the plane of the structures. Tensile deformation displacement is obvious and the expansion phenomenon is showed in the lozenge grid structure, which indicates that the structure is a typical negative breakage ratio structure. 
Table 3. Theoretical and numerical results.

\begin{tabular}{|c|c|c|c|}
\hline $\begin{array}{l}\text { Elastic } \\
\text { module }\end{array}$ & Theory(Pa) & $\operatorname{FEM}(\mathrm{Pa})$ & Error \\
\hline E11 & 2.84 E11 & 2.39E11 & 0.15 \\
\hline E22 & 3.42 E06 & 2.04E06 & 0.40 \\
\hline E33 & 1.34 E11 & $1.95 \mathrm{E} 11$ & 0.45 \\
\hline E12 & 0 & $1.52 \mathrm{E} 11$ & - \\
\hline E21 & 0 & $1.52 \mathrm{E} 11$ & \\
\hline E13 & 1.81 E11 & $1.52 \mathrm{E} 11$ & 0.16 \\
\hline E31 & 1.81 E11 & $1.52 \mathrm{E} 11$ & 0.16 \\
\hline E23 & 1.66 E11 & $1.52 \mathrm{E} 11$ & 0.08 \\
\hline E32 & 1.84 E11 & $1.52 \mathrm{E} 11$ & 0.17 \\
\hline
\end{tabular}

The theoretical results are compared with numerical data in Table 3. From this table we find favorable comparisons of our elastic constants (Young's modulus) are almost accordant. Note that some discrepancy is natural both due to the difference in the materials between the two cases and also from the different boundary conditions employed between the two FE models. Analytical closed-form formulas for square and hexagon based networks have been obtained, and the results are verified numerically. The equivalent modulus of elasticity of lozenge grid structure along $\mathrm{x}$ and $\mathrm{y}$ direction is obtained. However, the values of E12 and E21 are 0, and the moduli of elasticity in different forces are not zero. It shows that under the action of axial tension, the elastic deformation appears in the $\mathrm{X}$ direction, but shear effect appears in the $\mathrm{Y}$ direction and angular deformation appears. Under pure shear force, there is no elastic deformation but only shear behavior. From the data, the elastic modulus obtained by finite element method showed that the vascular stent exhibited elastic deformation under tension and shear, and the elastic modulus in $\mathrm{X}$ and $\mathrm{Y}$ direction was shown in the table. The results show that the theoretical Young's modulus is smaller than the elastic modulus calculated by the finite element method, but the overall trend is the same, the error is almost about $10 \%$. It shows that the method of calculating the equivalent Young's modulus based on energy conservation is feasible. The energy conservation method provides a new method for the analysis of structure elastic deformation.

\section{Conclusions}

In this work, a lozenge grid structure is proposed and material equivalent isotropic elastic modulus has been deducted with beam theory. Then structural deformation is carried out by finite element method by COMSOL Multiphysics. By comparing the theoretical formulas with the finite element simulation results, it is found that:

1. A new closed-form Young's modulus formulas is deduced by the energy principle, the elastic modulus of cellular structures under tensile and shear deformation is derive, and the formula for calculating Young's modulus is given.

2. Finite element analysis of the tension deformation between the lozenge grid structure and the simulation results and the error between them is not large, the equivalent elastic modulus is good associated with the Finite element results.
The analysis presented in this work provides a new method to analysis and calculate structural elastic deformation, and provided a new type sandwich structures unit cell.

Negative Poisson's ratio structure has a board application in the field of intelligent structure design and manufacturing in the future. It is worthwhile to study and guide the development and design of negative Poisson's ratio structures with advanced calculation methods and theories.

\section{References}

1. Davood M, Babak H, Ranajay G,Abdel M, Hamouda, Hamid $\mathrm{N}$ and Ashkan V. Theor. and Appl. J. Mech. L 681 (2016)

2. Etave F, Finet G, Boivin M et al. J. Bio. 341065 (2001)

3. Migliavacca F, Petrini L, Colombo M et al. J. Bio. 35803 (2002)

4. Migliavacca F, Petrini L, Valeria M et al. J. Med. Eng. and Phy. 2713 (2005)

5. Chua S N D, MacDonald B J, Hashmi M S J. J. Mat. Proc. Tech. 143591 (2003)

6. W Q, Liang D K, Yang D Z. J. Bio. 3921 (2006)

7. Xia Z, Ju F, Sasaki K. J. FE in Analy. and Design, 43649 (2007)

8. Petrini L, Migliavacca F, et al. Summer Bioengineering Conference Key Biscayne, Florida, 251 (2003)

9. Dumoulin C, Cochelin B. J. Bio. 33 1461(2000)

10. Stolpmann J, Brauer H, Stracke H J et al. J. Material wissensehaft and Werkstofftechnik 34736 (2003)

11. Perry M., Oktay S. and Muskivitch J.C. J. Min Invas Ther \& Allied Tech. 11165 (2002)

\section{Acknowledgments}

This report was funded by the Chongqing Nature Science Research Fund Program. The statements herein are solely the responsibility of the authors. 